\title{
Assessment of oxygen saturation levels during a mild hyperbaric chamber treatment
}

\author{
Gonzalez KE ${ }^{1}$, Hawkins JR ${ }^{1 *}$, Smith GA ${ }^{1}$, Heumann $\mathrm{KJ}^{1}$ and Potochny $\mathrm{NS}^{2}$ \\ ${ }^{1}$ Colorado Mesa University, Grand Junction, CO, USA \\ ${ }^{2}$ Veterans Affairs Medical Center, Grand Junction, CO, USA
}

\begin{abstract}
Purpose: Mild hyperbaric oxygen therapy is purported to increase healing by delivering higher concentrations of oxygen to damaged tissues secondary to an increase in atmospheric pressure. It is uncertain how oxygen saturation levels change during the course of a treatment. Our objective was to assess oxygen saturation levels during a treatment with and without atmospheric pressure and supplemental oxygen.
\end{abstract}

Methods: Ten participants received treatment in an OxyHealth mild hyperbaric chamber for 63 minutes in which the chamber settings were changed every 10 minutes. We measured participant's oxygen saturation levels every minute that they were in the chamber.

Results: Within subject effects were observed $(P \leq .001)$. It was concluded that chamber settings at 1.3 atmospheres absolute and supplemental oxygen gave way to the highest levels of oxygen saturation.

Conclusion: With this information clinicians can choose appropriate chamber parameters that provide the greatest increase in oxygen saturation and therefore the best clinical outcomes for tissue healing.

\section{Introduction}

The intent of hyperbaric oxygen therapy (HBOT) is to increase the oxygenation of a person's blood by forcing additional oxygen to dissolve into the blood plasma. This is done by increasing the atmospheric pressure within the chamber and therefore increasing the amount of oxygen available to the cells of the body [1]. These increases are hypothesized to lead to quicker cellular growth and repair [2]. During HBOT treatment, patients are put into a hyperbaric chamber where they breathe $100 \%$ oxygen while experiencing an increase in atmospheric pressure. Pressures are two to three times that of normal atmospheric pressure. With normal atmospheric pressure being $157 \mathrm{mmHg}$, the increases reached within the chambers can range from $988 \mathrm{mmHg}$ to $1,824 \mathrm{mmHg}$ [3]. Specific treatment settings are responsible for this range in pressure. For example, atmospheric pressure that reaches $1,824 \mathrm{mmHg}$ is done by using HBOT set at 2.4 atmospheres absolute (ATA) while patients breathe $100 \%$ oxygen. In addition to normal HBOT, there is also mild HBOT which is a treatment that uses less ATA, but theoretically continues to load red blood cells and plasma with oxygen. Mild HBOT can reach $988 \mathrm{mmHg}$ by using 1.3 ATA while breathing $100 \%$ oxygen.

In recent years the use of mild HBOT, also known as low pressure hyperbaric therapy, has grown. Applications include treatment of sports injuries, cerebral palsy, brain and head injuries, dermatological conditions, stroke, and more $[1,4,5]$. The increase in atmospheric pressure and oxygen saturation in the blood, during mild HBOT, is the foundation of thought that HBOT can lead to treatment of these conditions and ultimately quicker tissue repair.

In the basic steps of tissue repair, oxygen is a key component to tissue healing. For example, during collagenization, collagen fibers form the primary foundation for ligament, tendons, and scar tissue [6].
Excess amounts of oxygen are required to build the collagen fibers and to provide energy for the repair. The amount of collagen being formed in injured tissues is linearly related to the amount of oxygen available to the injured tissue [6]. Theoretically, placing a patient in a hyperbaric chamber should increase oxygen levels in the blood and therefore expedite the rate of tissue healing.

In reviewing HBOT literature, we found no studies that mention oxygen saturation measurements being obtained, although all claim that the positive effects reached by HBOT are due to the increase in oxygen levels. For example, one of the leading manufacturers of hyperbaric chambers claim that "HBOT saturates the oxygen in the blood," but provide no information on the extent of the oxygen saturation or at what atmospheric pressure blood is saturated [7]. To further illustrate this point, Golden et al. [1] stated, "There is a need to study the degree to which the factors of higher pressure and higher oxygen levels are responsible for the effect seen in this study. We must determine if it is the higher oxygen levels or the higher pressure or a combination of the two is responsible for the effects seen." This was further verified by Barata et al. [4] who stated, "With regards to HBOT, it is still necessary to determine the optimal conditions for these orthopedic indications, such as the atmosphere pressure, the duration of sessions, the frequency of sessions and the duration of treatment."

${ }^{\star}$ Correspondence to: Jeremy Hawkins, Colorado Mesa University, 1100 North Avenue, Grand Junction, CO 81501, USA, Tel: 970-248-1374; E-mail: jrhawkins@coloradomesa.edu

Key words: tissue healing, injury management, therapeutic modalities

Received: August 09, 2018; Accepted: August 20, 2018; Published: August 23 2018 
To this end, the purpose of this study was to observe a patient's percent oxygen saturation during mild HBOT treatment and to watch the changes in these levels as we altered the atmospheric pressure as well as the supplemental oxygen provided. It was hypothesized that with an increase in atmospheric pressure plus supplemental oxygen, blood oxygen saturation levels would increase to near $100 \%$ saturation.

\section{Methods}

This study was a cross-sectional design in which data was collected through noninvasive procedures routinely employed in clinical practice. The study was conducted in a human performance lab. All subjects were recruited via a classroom announcement and were encouraged to email or contact the investigator to seek further involvement. All subjects signed written consent forms, as approved by the Institutional Review Board. Subjects were also evaluated regarding their previous health history by a pre-participation questionnaire. Subjects completed an inclusion criteria questionnaire before participation. This study received appropriate approvals and began in October of 2014.

\section{Subjects}

Ten individuals ( 5 males, 5 females; height $=172.1 \pm 7.3 \mathrm{~cm}$, mass $=$ $70.4 \pm 10.6 \mathrm{~kg}$, age $=21.7 \pm 2.1 \mathrm{yrs}$ ) completed this study. One additional participant was unable to complete the study due to an increase in middle ear pressure. This participant was excluded from the study and replaced with another participant. All subjects were recreationally active individuals (defined as exercising less than 5 days a week) although not to the extent of an elite population of athletes (defined as exercising 5 or more days a week). This group corresponded with the athletic population that commonly uses the mild hyperbaric chamber clinically.

\section{Instruments}

This study was completed using a Vitaeris ${ }^{320}$ hyperbaric chamber (OxyHealth LLC, Santé Fe Springs, CA) with oxygen supplied by a SeQual Integra Ten Oxygen Concentrator (SeQual Technologies, Inc., San Diego, CA). Participants wore an AMSure oxygen mask (Amsino International, Inc., Pomona, CA) to facilitate oxygen delivery. Oxygen saturation was measured with BCI 3301 hand-held pulse oximeter (Smiths Medical PM, Inc., Waukesha, WI).

\section{Interventions}

Participants received treatment in a Vitaeris ${ }^{320}$ OxyHealth mild Hyperbaric Chamber for 63 minutes. Treatment order was either 1) 10 minute at no pressure and no oxygen supplementation; 2) 10 minute at 1.3 ATA and no oxygen supplementation; 3) 10 minute at 1.3 ATA with oxygen supplementation; 4) 10 minute at no with oxygen supplementation; and 5) 10 minute at no pressure and no oxygen supplementation, or the exact opposite order. Time to change pressure accounted for the remainder of the time.

Participant's oxygen saturation levels were recorded every minute while in the chamber using a pulse oximeter. The pulse oximeter was worn on the right index finger. Oxygen was delivered at the specific intervals via a non-rebreather mask at $10 \mathrm{~L} / \mathrm{min}$ using an oxygen saturation machine. The non-rebreather mask allowed a person to breathe approximately $55 \%$ pure oxygen. After participating, participants were given take home care instructions and were instructed to contact the investigator if any abnormal changes in health arose.

\section{Statistical analysis}

Oxygen saturation to the nearest percent was the main outcome measure. Data was analyzed using a repeated measures ANOVA followed by multiple pairwise comparisons to determine the treatment effect (IBM SPSS Statistics version 21.0, New York).

\section{Results}

A within subject difference was observed $\left(\mathrm{F}_{3,27}=22.767, P \leq .001\right)$ (Figure 1). Oxygen saturation levels for no pressure and no oxygen supplementation $(96.63 \pm 1.31 \mathrm{mg} / \mathrm{L})$ differed from 1.3 ATA pressure and no oxygen supplementation $(97.91 \pm .58 \mathrm{mg} / \mathrm{L}, P=.002), 1.3$ ATA with oxygen supplementation $(98.53 \pm .44 \mathrm{mg} / \mathrm{L}, P=.001)$, and no pressure with oxygen supplementation $(98.66 \pm .57 \mathrm{mg} / \mathrm{L}, P=.001)$. The 1.3 ATA pressure and no oxygen supplementation differed from the 1.3 ATA with oxygen supplementation $(P=.005)$. The 1.3 ATA pressure and no oxygen differed from no pressure with oxygen supplementation $(P=.001)$. The treatments with oxygen supplementation did not differ from one another $(P=.328)$. The treatment order made no difference in the results $\left(\mathrm{F}_{1,8}=.546, P=.481\right)($ Table 1$)$.

\section{Discussion}

In this study, it was hypothesized that during a mild HBOT at 1.3 ATA with oxygen supplementation oxygen saturation levels would increase to near $100 \%$. This was observed to be true. Interestingly, although 1.3 ATA and supplemental oxygen increased oxygen saturation the most, it was not the only settings that brought oxygen saturation levels to near $100 \%$. Oxygen supplementation alone also increased to this level. Although there continued to be rises in oxygen saturation percentages with increased atmospheric pressures alone, the increases were not as significant as those that included supplemental oxygen. Results were consistent regardless of treatment order. Based on the results, the level of atmospheric pressure did make a difference in oxygen saturation percentages in comparison to the other settings.

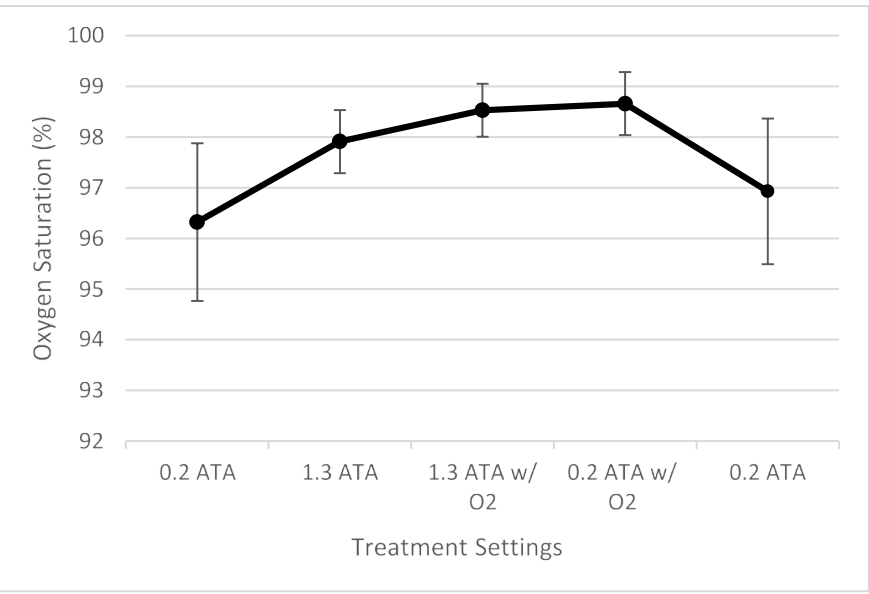

Figure 1. Oxygen saturation concentrations over the time course of the treatment

Table 1. Average Oxygen Saturation Percentages during Treatment $(1=.2$ ATA, $2=1.3$ ATA, $3=1.3$ ATA with oxygen supplementation, $4=.2$ ATA with oxygen supplementation)

\begin{tabular}{|c|c|c|c|c|c|}
\hline Time (min) & $5: 00-15: 00$ & $18: 00-28: 00$ & $28: 00-38: 00$ & $43: 00-53: 00$ & $53: 00-63: 00$ \\
\hline $\begin{array}{c}\text { Treatment } \\
\text { Order }\end{array}$ & 1 & 2 & 3 & 4 & 1 \\
\hline $\begin{array}{c}\text { Subject } \\
\text { Average } \\
\text { (3M, 2F) }\end{array}$ & $95.70 \%$ & $97.60 \%$ & $98.70 \%$ & $98.60 \%$ & $96.80 \%$ \\
\hline $\begin{array}{c}\text { Treatment } \\
\text { Order }\end{array}$ & 1 & 4 & 3 & 2 & 1 \\
\hline $\begin{array}{c}\text { Subject } \\
\text { Average } \\
\text { (2M, 3F) }\end{array}$ & $97.10 \%$ & $98.70 \%$ & $98.40 \%$ & $98.20 \%$ & $96.90 \%$ \\
\hline
\end{tabular}


In current related research studies, treatment parameters include a placebo setting which is typically set at 1.3 ATA, and a treatment group that typically receives 2.0 ATA. The outcome of many of these studies conclude that the benefits obtained from HBOT were the same regardless of the placebo setting or the treatment group setting. To reach oxygen saturation levels nearest $100 \%$ it can be concluded that a 1.3 ATA with supplemental oxygen treatment can prove to have the greatest increases in oxygen saturation percentages.

The use of hyperbaric chambers in sport is growing. Some athletes purchase portable hyperbaric chambers to use as they train and compete. In regard to the results of this study, we question whether the use of a hyperbaric chamber alone is beneficial to the athlete. OxyHealth, LLC does not sale their hyperbaric chambers in conjunction with the supplemental oxygen machines. The oxygen concentration machines are available for purchase with the hyperbaric chamber but are not always utilized. We wonder if athletes who employ their hyperbaric chamber without an oxygen saturation machine are receiving much benefit.

A limitation to this study is that the treatments were given at specific intervals for a maximum of 10 minutes. Even though subjects received a 63 minute treatment, they did not receive the same treatment for 63 minutes. Typical mild HBOT sessions last for at least 60 minutes, but more commonly have a duration of 60-120 minutes. It is possible that a prolonged treatment time at a given setting would result in different data outcomes. In this study, percent oxygen saturation levels did not fluctuate after approximately 4 minutes at each setting, so we question whether they would change over a longer period of time.

\section{Clinical implication}

Mild HBOT is a good resource for clinicians. With the information obtained from this study we can see that mild HBOT at 1.3 ATA with oxygen supplementation brought on oxygen saturation levels nearest to $100 \%$. Although oxygen supplementation alone increased oxygen saturation levels to near equal results, there still proves to be a greater oxygen saturation at 1.3 ATA with oxygen supplementation. Additional measurements are needed to determine whether the increased pressure and oxygen supplementation has effect beyond what is measurable with a pulse oximeter. With this information clinicians can choose to use the appropriate chamber parameters that provide the greatest increase in oxygen saturation and therefore result in the best clinical outcomes for tissue healing.

\section{References}

1. Golden Z, Golden CJ, Neubauer RA (2006) Improving Neuropsychological Function After Chronic Brain Injury with Hyperbaric Oxygen. Disabil Rahabil. 28:1379-186. [Crossref]

2. Cifu DX, Hart BB, West SL, Walker W, Carne W (2014) The Effect of Hyperbaric Oxygen on Persistent Postconcussion Symptoms. J Head Trauma Rehabil. 29:11-20. [Crossref]

3. Hyperbaric Oxygen Therapy or "Mild" Hyperbaric therapy. Oxford Recovery Center Web site. http://oxfordhbot.com/hard-vs-soft-chambers/. Accessed February 16, 2015.

4. Barata P, Cervaens M, Resende, R, Camacho Ó, Marques F (2011) Hyperbaric Oxygen Effects on Sports Injuries. Ther Adv Musculoskelet Dis. 3:111-121. [Crossref]

5. Collet JP, Vanasse M, Marois P, Amar M, Goldberg J, et al. (2001) Hyperbaric Oxygen for Children with Cerebral Palsy: A Randomised Multicentre Trial. HBO-CP Research Group. Lancet. 357:582-586. [Crossref]

6. Knight KL, Draper DO. Therapeutic Modalities: The Art and Science. Baltimore, MD: Lippincott Williams \& Wilkins; 2008.

7. Hyperbaric Oxygen Therapy - HBOT: Hyperbaric Medicine. Oxyhealth LLC Web site http://www.oxyhealth.com/hbot-chambers.html. Accessed February 1, 2015.

Copyright: $\odot 2018$ Gonzalez KE. This is an open-access article distributed under the terms of the Creative Commons Attribution License, which permits unrestricted use, distribution, and reproduction in any medium, provided the original author and source are credited. 